\title{
Warum hört man so wenig wirklich gute Argumente für die Privatisierung des Strafvollzugs?
}

Heinz Cornel

$\mathrm{N}$ och vor der Frage der Verfassungsmäßigkeit und damit der rechtlichen Zulässigkeit der Privatisierung der Strafvollstreckung und insb. des Strafvollzugs ist die Frage zu stellen, warum dies eigentlich angestrebt werden sollte. Dazu genügen die allgemeinen Schlagworte vom schlanken Staat, der auf seine Kernaufgaben reduziert werden sollte, sicher nicht.

Es geht grundsätzlich um die Frage, nach welchen Grundsätzen und welchen Methoden eine Gesellschaft in jeweils ganz konkreten Bereichen gesteuert werden soll - Gemeinwohlorientierung mit demokratisch legitimierter Kontrolle oder Gewinnorientierung. Mit dieser Unterscheidung ist man jedoch nur einen kleinen Schritt weiter, weil einerseits behauptet wird, dass die gewinnorientierte Steuerung über den Markt zu einer optimalen Erfüllung des Gemeinwohls führt und andererseits Gemeinwohlorientierung noch nicht automatisch auch zur Erreichung dieses Ziels führt - ein großer Teil der Kritik an staatlichem Handeln und insbesondere an bürokratischen Prozessen lässt daran eher Zweifel aufkommen. Darüber hinaus gehende ethische Aspekte der Gerechtigkeit lasse ich hier unbeachtet.

Die veröffentlichte Meinung geht in den letzten Jahren - parallel zu einer Abkehr bzw. Zurückdrängung des sozialstaatlich-integrativen Gesellschaftsmodells, einer Ökonomisierung des Alltags und dem internationalen Triumphzug des Neoliberalismus - stark in Richtung Privatisierung und Deregulierung, befürwortet also eines der möglichen Steuerungsmodelle deutlich. Diese Präferenz sollte man in seiner Bedeutung aber nicht übergewichten, denn sie ist mit vielfältigen Interessen verwoben:

- Wer von der Politik als Betriebs- und Volkswirtschaftler zum Experten erklärt wird, hat die gewinnorientierte Steuerung über den Markt vom ersten Tag seines Studiums an als sein Handwerkszeug gelernt und so liegt es nahe, dass er davon ebenso überzeugt ist, wie der Strafrechtler, der das Strafrecht für das zentrale Instrument der Erzielung von Normtreue der Bevölkerung hält. Als Schiedsrichter eignet sich nicht, wer für eine Mannschaft allein die Spielregeln definieren will, denen er selbst verpflichtet ist.

- Das gleiche gilt für den, der allerlei Managementkurse belegt und besucht, weil er sich davon und von einer an der Betriebswirtschaft orientierten Sprache in allen Organisationen eine Verbesserung der Effektivität verspricht er hat eine akzeptable Entscheidung getroffen, die sich aber inhaltlich noch legitimieren und bewähren muss und nicht schon deshalb richtig ist, weil sie neu ist.

- Privatisierungen sind oft mit spezifischen Verdienstmöglichkeiten verbunden, die ein Plädoyer dafür nahe legen können. Da sind Aufsichtsräte und Beiräte zu besetzen (Bahn, Post und Telekom machten das vor) und der eine oder andere Experte und Politiker sieht sich für diese Aufgaben womöglich prädestiniert.

- Privatisierungen können langfristig dazu genutzt werden, Verantwortlichkeiten für politische Entscheidungen $\mathrm{zu}$ verschleiern, indem sie als allein von ökonomischen Sachzwängen geleitet dargestellt werden. Wo es keinen Entscheidungsspielraum gibt, wo Strukturen, Eigentumsverhältnisse und Steuerungsmethoden des Wirtschaftens quasi natur-, technik- oder gottgegeben sind, da lohnt kein Denken und Suchen nach Alternativen und keine politische Debatte. Dabei handelt es sich natürlich um einen Zirkelschluss, der den privatisierten Unternehmen nicht vorzuwerfen ist - wer ökonomische Kriterien für die allein bestimmenden erklärt, darf sich nicht wundern, wenn etwa durch Bahnstreckenstilllegungen ganze Regionen vom öffentlichen Nahverkehr abgekoppelt werden, Gehbehinderten Fahrpreisermäßigungen gestrichen und Briefkästen demontiert werden. Wer aber genau dies aus Spargründen vorhat, versteckt sich gerne hinter Sachzwängen und muss sich nicht in öffentlicher Debatte demokratisch rechtfertigen.

- Die politischen Entscheidungen für Privatisierungen beziehen für Politiker der Exekutive und Legislative einen besonderen Charme auch daraus, dass Kosten aus der Gegenwart in die Zukunft verlagert werden, ohne dass sie direkt als Neuverschuldung ausgewiesen werden müssen. Zwar wird man Gefängnisse sicher nicht verkaufen können, wie das bei Telekom, Post, Stadtwerken und ganzen U-Bahnsystemen möglich war, die über viele Jahrzehnte durch Steuerleistungen bezahlt worden waren und dann in einzelnen Jahren Milliarden zur Haushaltsentlastung beitrugen, immerhin aber müsste so ein Gefängnisneubau nicht in wenigen Jahren finanziert werden, sondern die zukünftigen (eventuell weit höheren) Kosten verteilen sich über Jahrzehnte. Wer für 4 Jahre ein Mandat hat und von Haushaltsjahr zu Haushaltsjahr hüpft mag das für rational halten - wer sich ein wenig kritische Distanz bewahrt hat sollte die Kosten vergleichen, die insgesamt in 20 oder 30 Jahren entstanden sind.
Alle genannten Personengruppen können ihre Argumente und Interessen in den Diskurs um die Privatisierung des Strafvollzugs, der Strafvollstreckung allgemein und auch der Straffälligenhilfe, die zum Teil ja längst privat organisiert ist, ${ }^{1}$ aber noch kaum gewinnorientiert gesteuert wird, einbringen. Wahrheitsbeweise sind solche Befürwortungen aber nun wirklich nicht.

Ein Argument der Privatisierungsbefürworter ist nachvollziehbar, aber keines, was gesamtgesellschaftlich ausschlaggebend sein kann: Es wird mit fast allem Geld verdient, von Industrie- und Lebensmittelproduktion über den Handel mit Kriegswaffen und Schuldtiteln bis zur Prostitution - warum sollte nicht auch mit dem Einsperren von Menschen Geld verdient werden? Man braucht nur jemanden, der einen dafür bezahlt und solche Menschen lassen sich mit guter Werbung finden. ${ }^{2}$ Aber so einfach wollen wir es uns nicht machen - Argumente und Kriterien können durchaus benannt werden - sie müssen aber zumindest an empirische Erfahrungen anknüpfen und in gleicher Weise für den staatlichen und möglicherweise privatisierten Strafvollzug angewendet werden.

Es geht dabei auch nicht darum, ein an sich unhinterfragbares Erfolgsmodell einfach nur zu übertragen. So eindeutig ist die Bilanz nicht schon der einfache Zeitungsleser ist durchaus in der Lage, Fragen zu stellen und muss sich nicht an die Devise im Märchen von des Kaisers neue Kleider halten:

- Von den Fesseln behördlicher Strukturen befreit hat die Berliner Bankgesellschaft sich ganz auf die Marktmechanismen beim Geldverdienen verlassen. Das eingesetzte Geld kam vom Steuerzahler und in wenigen Jahren wurde damit so erfolgreich privat gewirtschaftet, dass die Schäden für den Berliner Landeshaushalt erheblicher sind als die Folgen angeblich überdimensionierter überteuerter Öffentlicher Dienste aufgrund der Teilung. Dass dabei eine Regierungspartei noch mit Spenden bedacht wurde, ist fast schon nebensächlich. Jugendliche in heruntergekommenen, seit neuestem privat (mehr oder weniger) geputzten Schulen und Kinder, denen aus Spargründen das Dessert in der KiTa gestrichen wurde, werden sicher die Risikobereitschaft derjenigen bewundern, die nun trotz der von Ihnen verursachten Milliardenverluste (die Schätzungen des Berliner Senats schwanken zwischen 21,6 und 35 Milliarden) hohe Abfindungen, Dienstwagen und teils 
sogar Schadenersatzleistungen in Millionenhöhe als Geschädigte erhalten. Mit monatlichen Pensionen von 19 812,56 Euro bis 30000 Euro pro Person und allerlei Vergünstigungen und Serviceleistungen wird immerhin etwas für die Belebung des danieder liegenden Berliner Konsums getan.

- Die Verwaltungskosten der deutschen gesetzlichen Rentenversicherung mit den jeweils persönlich erworbenen Rentenansprüchen, wie sie in vielen Ländern der Welt angestrebt werden, betragen etwa 1,6\% der Gesamtkosten - bei den privaten Lebensversicherungen sind es 3,5\% bis 6,5\% (einschließlich der notwendigen Kapitalanlagekosten). Man wundert sich nicht, dass der Ehrenvorsitzende einer Partei, die sich für die Privatisierung der Rentenversicherung ausspricht, mehrere Aufsichtsratmandate in der Versicherungswirtschaft hat. Der Chef des französischen Versicherungskonzerns Axa brachte die unterschiedliche Perspektive im Jahr 2000 in einem Interview mit Le Monde (18.2.2000) angesichts einer beabsichtigten Verdoppelung der Prämien für Behindertenrenten mit den Worten auf den Punkt: »Ich bin eine Versicherungsgesellschaft, mir geht es um Gewinn, nicht um Solidarität. « In Chile wurde das Pensionssystem übrigens komplett privatisiert - die Verwaltungskosten betragen nun 30\% der eingezahlten Beiträge.

- Wie gelungen war der Start des LKW-Mautsystems und welche Erfahrungen ergeben sich daraus für die Partnerschaft beim Aushandeln von Verträgen sowie für das Tragen der Risiken? Welche privaten Unternehmen können für Milliardenrisiken solide bürgen und wie gleichberechtigt verhandeln hoch spezialisierte Anwaltsbüros mit Millionenhonoraren (die zu den Kosten gehören!) mit Ministerialbeamten?

- Am 8. September 2005 trat das »Gesetz zur Beschleunigung der Umsetzung von Öffentlich Privaten Partnerschaften und zur Verbesserung gesetzlicher Rahmenbedingungen für Öffentlich Private Partnerschaften « in Kraft. Welche Lehren werden gezogen aus der Public-PrivatePartnership hinsichtlich des Olympia-Stadions in Berlin, wo schon nach wenigen Jahren der eine private Partner Insolvenz anmeldete und dem anderen privaten Partner die Hälfte der Stadionmiete erlassen werden musste. Die Kosten vom mittelfristig 50 Millionen Euro tragen die Steuerzahler - vor Tische las man es anders.

- In NRW sind die Müllgebühren in dem Kreis heute am niedrigsten, der als einer der wenigen seine Stadtreinigung nicht privatisierte, sondern in kommunaler Trägerschaft weiter betreibt. Immerhin verfügen aber zahlreiche Geschäftsführer privatisierter Müllbetriebe nach dutzenden Müllskandalen und Korruptionsaffären in vielen nordrheinwestfälischen Großstädten über ausreichend Hafterfahrung, um private Gefängniskonzerne mit Insiderwissen beratend unterstützen zu können.

- Zehntausende Münsterländer hatten Ende November 2005 Gelegenheit, mehrere Tage im Dun- keln darüber nachzudenken, ob die RWE von ihrem Gewinn in Höhe von 4,7 Milliarden Euro in den ersten Monaten des Jahres 2005 nicht ein wenig hätte in Strommasten investieren können, von denen viele älter als 60 Jahre sind und über die seit fast 40 Jahren bekannt ist, dass sie aus einem Stahl bestehen, der eben leicht einknickt. Der Berliner Tagesspiegel schrieb dazu: »Die Gewinne scheinen privatisiert, während die Kosten von möglichen Schäden auf die Allgemeinheit abgewälzt werden. So weigert sich RWE, Schadenersatzforderungen von betroffenen Bürgern und Unternehmen anzuerkennen.«(Tagespiegel 6.12.2005, S. 10). Der Zeit vom 8.12.2005 (S. 30) konnte man entnehmen, dass »seit der Liberalisierung des Strommarktes im Jahr 1998 die Investitionen der Netzbetreiber kontinuierlich von etwa 4 Milliarden Euro auf rund 2 Milliarden Euro im Jahr 2004 gesunken (sind) - bezogen auf die reinen Materialkosten ... Die Stromkonzerne seien längst dazu übergegangen, regelmäßige Wartungszyklen durch ,zustandsorientierte' Wartungsarbeiten zu ersetzen.« Dieses Problem ist übrigens nicht neu, weil in Schweden nach der Privatisierung im Winter 2001 zehntausende Haushalte tagelang ohne Strom waren, weil die Reparaturtrupps für geknickte Leitungen eingespart worden waren und in Kalifornien es seit der Privatisierung vor 10 Jahren ständig mehrfach im Jahr zu Stromausfällen kommt.

- Die ehemals staatliche Bundesdruckerei Berlin, in der unter anderem Banknoten und Personalausweise gedruckt werden, wurde zunächst verkauft, ging aber kurz darauf mit 500 Millionen Euro Schulden in die Pleite. Da es aber einen unabweisbaren Bedarf an der Herstellung von Personalausweisen und Banknoten gab und gibt (man wollte das wohl nicht ganz dem Markt überlassen) sanierte der Bund mit öffentlichen Geldern die Druckerei und verkaufte sie wiederum an einen privaten Betreiber zu einem symbolischen Preis von 1 Euro. Mehr als 2000 Drucker verloren im Zuge dieses Privatisierungsprozesses ihren Arbeitsplatz.

- In Berlin wurden 1998 die Gaswerke für 721 Millionen Euro verkauft. Kurz darauf wurde die Hälfte der Arbeitsplätze (1381 von 2563) abgebaut, die Preise stark erhöht und das Gasnetz, also nur ein Teil des ganzen Kuchens, für 818 Millionen an eine Leasing Firma weiterverkauft. Die Wasserbetriebe wurden im Folgejahr teilprivatisiert, die Instandhaltungskosten sofort auf die Hälfte reduziert und die Verbraucherpreise stark erhöht.

Man kann diese Erfahrungen sicher relativieren, auch wenn dem Leser und der Leserin nicht entgangen sein dürfte, dass der allgemeinen Privatisierungseuphorie eine gewisse Skepsis entgegen gesetzt werden soll. Es soll gezeigt werden, dass die Privatisierungsideologie nicht voraussetzungslos und unhinterfragbar ist. Privatisierungen sind nicht immer Erfolgsgeschichten - es gibt bekanntlich auch Missmanagement, Insol- venzen und Situationen, in denen die öffentliche Hand nochmals nachschießen muss, um öffentliche Aufgaben zu erfüllen - bekanntlich finden Gewinne immer einen Adressaten und Eigentümer und Verluste werden oft letztendlich sozialisiert. Das Marktmodell findet eben eine Grenze dort, wo der Kunde nicht einfach auf das Produkt und seinen Anbieter verzichten kann, zumal es oft monopolisiert ist - wie z. B. bei der Wasser- und Energieversorgung, der Verkehrsanbindung, vielleicht auch bei Gefängnissen.

Dies ist auch deshalb ein Problem, weil nach einer negativen Erfolgskontrolle der Privatisierung es regelmäßig keinen Weg zurück gibt - oder aber nur einen mit sehr hohen Hürden bzw. erst nach 30 und mehr Jahren. Großbritannien hat nach 10 Jahren die Ergebnisse der Privatisierung von British Rail ausgewertet. Nach der Privatisierung erhöhten sich die Subventionen für den Schienenbetrieb zwischen 1996 und 2001 mit 19 Milliarden Euro auf den höchsten Betrag aller Zeiten Kosten wurden also nicht für den Steuerzahler eingespart, aber Zuverlässigkeit und Sicherheit sanken unbestreitbar erheblich. Die für das Schienennetz verantwortliche Railtrack kündigte 2001 ihren Aktionären eine Dividende von 138 Millionen Pfund an - kurz danach musste sie Konkurs anmelden. Inzwischen rechnet man mit einem Investitionsbedarf von 100 Milliarden Euro. Als Konsequenz möchte die Regierungspartei die Privatisierung rückgängig machen - wahrscheinlich noch vor dem Börsengang der Deutschen Bahn.

Eine Wirkungsforschung mit vergleichbaren Bedingungen und offenem Ergebnis, aus der man dann Konsequenzen in die eine oder andere Richtung ziehen kann, findet nicht statt.

Die Stimmung in der Bevölkerung gegenüber den Privatisierungen ist entgegen der Meinung vieler Experten und Politiker sehr viel reservierter kein Wunder, denn viele der oben genannten Interessen treffen auf die Bevölkerung nicht zu.

Zahlreiche Bürgerentscheide und Bürgerbegehren waren in den letzten Jahren erfolgreich beispielsweise gegen die Privatisierung ihrer Stadtwerke (z. B. in Düsseldorf und Münster), der Wasserversorgung (allein 7 in Bayern), der Stromversorgung (z. B. in Erlangen), der Müllabfuhr oder von Kliniken. In Hamburg hat der Senat den Bürgerwillen schlicht ignoriert.

Mit Staatsferne im Sinne von Zurückdrängung des Obrigkeitsstaats und mehr demokratischer Mitbestimmung und Selbstverwaltung hat die Politik der Privatisierung ohnehin nichts zu tun - im Bereich des Strafvollzugs wohl am allerwenigsten. Der Anteil öffentlicher Ausgaben der Städte, Gemeinden, Landkreise, Länder und des Bundes bezogen auf das Bruttoinlandsprodukt (die so genannte Staatsquote) ist im übrigen in den letzten 30 Jahren stark von über $31 \%$ auf $26 \%$ gesunken. Die Ausgaben der Sozialversicherungen sind zwar 
gestiegen, weil die Arbeitslosigkeit stieg und die Menschen länger leben. Insgesamt bleibt es aber auch beim Hinzurechnen der Sozialversicherungsausgaben - entgegen dem Eindruck in der öffentlichen Debatte - bei einem Sinken der Staatsquote seit 1975 (vgl. Bundesministerium der Finanzen, Bundeshaushalt 2004, Tabellen und Übersichten, November 2003, S.24).

Die Argumente, die sich ganz konkret auf die Privatisierung des Strafvollzugs oder seiner Teile beziehen, stellen oft die Kostenvorteile in den Vordergrund - meist sind es erhoffte Vorteile, denn die Fachliteratur ist zum einen sehr skeptisch und bescheiden ${ }^{3}$ und zum anderen darf man sich natürlich im Kostenvergleich nicht auf Teilsysteme beschränken, ohne die Nebenfolgen zu bedenken. Das darf rein rechtlich ein Betriebswirtschaftler, der als Vorstandschef eines Unternehmens Lücken im Umwelt- und Steuerrecht nutzt, Steueroasen aufsucht, Endfünfziger kurzarbeiten lässt und hoch qualifizierte Wissenschaftler als kostenlose Praktikanten beschäftigt oder in einem hochprofitablen Unternehmen Tausende entlässt, deren Arbeitslosigkeit dann öffentlich finanziert wird - in einem seriösen wissenschaftlichen Kostenvergleich wird man solche Verfahren nicht für angemessen halten.

Kostenvergleiche müssen vergleichbare Kosten vergleichen und dürfen nicht willkürlich tatsächlich vorhandene Kosten ausblenden:

1. Hinsichtlich der Löhne müssen gleich qualifizierte Mitarbeiter in vergleichbaren Beschäftigungsverhältnissen verglichen werden. Erreichte und gesellschaftliche gewünschte Standards der arbeitsrechtlichen und sozialen Absicherung sowie der Mitarbeitermitbestimmung (Personalvertretung) und beispielsweise der Beschäftigung von Behinderten dürfen nicht als reine Kostenfaktoren untergraben werden - jedenfalls wäre der Kostenvergleich dann nicht seriös. In den USA wurde in den privaten Anstalten ein erheblich niedrigeres Ausbildungsniveau der Bediensteten und eine höhere Fluktuation festgestellt (vgl. Gasch 2004, S. 268).

2. Des Weiteren ist zu prüfen, ob die Leistungen in gleicher (oder besserer) Qualität angeboten werden und weshalb sich - gegebenenfalls Kostenvorteile ergeben. Wer beispielsweise die Ergebnisse der Privatisierung der Reinigungsdienste an Berliner Schulen betrachtet, der wird nicht nur das Aussteigen aus tariflichen Vereinbarungen für den öffentlichen Dienst bemerken, sondern auch den ständigen Wechsel des Personals, das sich meist mit osteuropäischen Sprachen verständigt, und einen erheblich niedrigeren Qualitätsstandard. Das Personal in diesen Putzkolonnen muss unter schlechtesten, ungesicherten Arbeitsbedingungen mehr leisten, als möglich ist. Nebenleistungen, wie z.B. eine regelmäßige Ansprechbarkeit für Schüler oder Unterstützung beim Wiederauffinden von ver- gessenem Schulmaterial oder Kleidung ist da unmöglich. Mit Angstschweiß wischt es sich offensichtlich schneller, aber nicht besser. Maelicke, der Privatisierungen im Bereich des Strafvollzugs durchaus positiv gegenübersteht, hat die Risiken früh und grundsätzlich beschrieben: »Profitorientierung zielt nach den Gesetzen der (sozialen) Marktwirtschaft auf Profitmaximierung. Maximierung wird erreicht bei einer möglichst großen Differenz zugunsten des Anbieters zwischen den Kosten für die Dienstleistung und dem erzielten Preis. Deshalb besteht unter rein kommerziellen Interessen die Gefahr der Leistungsabsenkung, um möglichst einen hohen Gewinn zu erzielen. ${ }^{4}$

3. Ein Kostenvergleich muss alle Folgen und Nebenfolgen einbeziehen, die sich aus der öffentlichen oder privaten Organisationsform ergeben. Betriebswirtschaft genügt da eben nicht man kann nicht die alte staatliche Kameralistik kritisieren, um dann selbst nur ein Teilsystem zu betrachten. Wer beispielsweise die Lebensmittelversorgung und Ernährung der Gefangenen aus Kostengründen privatisiert, muss auch bedenken und gegebenenfalls berechnen, was das für die Beschäftigung der Gefangenen bedeutet, die ansonsten in der Anstaltsküche tätig waren. Wird durch schlecht ausgebildetes Personal nicht entsprechend dem Vollzugsziel und den Grundsätzen über die Gestaltung des Vollzugs gehandelt oder können diese nicht gem. $\S 154$ StVollzG mitwirken, die Aufgaben des Vollzugs zu erfüllen, dann hat man zwar für isoliert betrachtete Tätigkeiten gespart, insgesamt aber für Folgekosten gesorgt.

4. Mögliche Insolvenzen der privaten Anbieter müssen in die Kostenkalkulation eingerechnet werden, denn im entsprechenden Fall können Vollzugsanstalten nicht einfach zur Konkursmasse gezählt, sondern müssen von der öffentlichen Hand weiter betrieben werden (wie das Olympiastadion in Berlin). Man mag beispielsweise die Insolvenz des Immobilienspekulanten Schneider in den 90er Jahren, dessen Geschäfte im Zusammenwirken mit der Deutschen Bank zu einem Schaden von etwa 6 Milliarden DM führte ${ }^{5}$, als bedauerlichen Unfall eines an sich funktionierenden Systems bezeichnen ${ }^{6}$ - aber man wird die faktische Enteignung beispielsweise von Tausenden Handwerkern und Arbeitnehmern aus der Gesamtrechnung über die Effektivität dieses Systems nicht einfach unberücksichtigt lassen können. Die britische Railtrack und das Berliner Olympiastadion belegen auch, dass das Risiko bei Privatisierungen so gering nicht ist.

5. Schließlich müssen die Kosten für die Leistungskontrolle des Staates über das Erbringen der Leistungen durch Private, die Vertragsverhandlungen und Ausschreibungen, Verfahren bei Schlechtleistungen usw. selbstverständlich in die Kostenrechnung einfließen, denn sie sind Folgen einer privatisierten Leistungserbringung.

Wer die (Teil-)Privatisierung eines Gefängnisses zum Leuchtturm seiner Politik erklärt (so der hessische Ministerpräsident Koch bei der Einweihung der JVA Hünfeld laut Tagesspiegel vom 6.12.2005, S.6), der wird sicher erklären können, warum die Betreiberfirma Serco durch den Einsatz ihrer 95 Mitarbeiter angeblich 660000 Euro pro Jahr sparen kann.

- Werden die privaten Mitarbeiter wesentlich schlechter bezahlt, so wird der Ministerpräsident den MitarbeiterInnen des Allgemeinen Vollzugsdienstes oder der Fachdienste landesweit erklären müssen, dass er der Meinung ist, sie seien wesentlich zu gut bezahlt.

- Werden die privaten Mitarbeiter - ähnlich wie die schwarzen Sheriffs im Vergleich zur Polizei weniger und kürzer ausgebildet und er meint, dass das auch ausreicht, dann wird er der erstaunten Öffentlichkeit erklären müssen, warum er öffentlich Bedienstete unnötig lang und gut ausbildet.

- Werden die privaten Mitarbeiter schließlich nach Meinung der Landesregierung Hessen effektiver eingesetzt, so wird der Justizminister erklären müssen, warum er gut oder gar besser ausgebildete Mitarbeiter des öffentlichen Dienstes weniger effektiv einsetzt und dadurch landesweit Steuermittel verschleudert - es geht schließlich um seinen Verantwortungsbereich, denn Minister und Ministerpräsidenten sind immer noch öffentlich bezahlte Träger von Verantwortung und nicht private Manager.

Immerhin gibt es auch Innovationen privater Betreiber, die weltweit Schule machen sollten und die Pönologie sollte sich selbstkritisch fragen, warum dies nicht längst zum Mindeststandard des Strafvollzugs gehört. In der JVA Hünfeld sollen Neuankömmlinge mit einer Tasse Kaffee empfangen werden, weil man um die »befriedende Wirkung freundlicher Behandlung « wisse (vgl. Tagespiegel vom 6.12.2005, S. 6). Da wird die Inhaftierung endlich zum attraktiven Event. » Hier Serco - was kann ich für Sie tun?«.

All dies spricht noch nicht gegen Privatisierung, möchte aber das Schweigen über Probleme, die Einseitigkeit und unreflektierte Umgehensweise beenden sowie ein Niveau einer Debatte einfordern, in der wirklich Erfahrungen und nicht nur Teilinteressen eine Rolle spielen.

Als Argumente für die Privatisierung werden nicht nur wirtschaftliche genannt und Aspekte der demokratischen Kontrolle und der Verfassungsmäßigkeit hintangestellt, es werden auch gerne Vorurteile hinsichtlich öffentlicher Verwaltung bedient (bis in persönliche Diffamierungen der Beamten an sich, denen bestimmte Charakterund Arbeitseigenschaften zugeschrieben werden), 
Bürokratiekritik angesprochen und gar Staatsferne und Entstaatlichung beschworen. Letzeres ist natürlich schon deshalb pikant, weil der Strafvollzug ein ganz besonderes Zeichen des staatlichen Gewaltmonopols ist, und auch im privaten Gefängnis der Gefangene nicht zum König Kunden wird, der das Angebot ausschlagen kann oder lieber etwas weniger Fluchtsicherung bestellt. ${ }^{7}$

Letztlich geht es in der Debatte auch um ein Menschenbild. Es wird vermittelt, dass Menschen grundsätzlich nur durch ständige Leistungsanreize und Angst vor Arbeitsplatzverlust zu motivieren sind - und dass dies eben im öffentlichen Dienst nicht möglich sei. Dass man dazu Anlässe für Kritik findet, ist selbstverständlich und es ist nicht im Geringsten im Sinne dieser Zeilen Veränderungsbedarf zu leugnen. Das Ausnutzen von Arbeitsplatzsicherheit gibt es ebenso, wie man es vielleicht als Fehlsteuerung bezeichnen darf, wenn Manager im Nachhinein für die Nichterreichung des erklärten Geschäftsziels Erhaltung der Selbstständigkeit des Unternehmens 30 Millionen Euro Gratifikation bekommen. Ein Beamter muss schon ziemlich oft »krankfeiern« um gesellschaftlich und wirtschaftlich von seinem Fehlverhalten ähnlich zu profitieren. Es kann und soll also gar nicht bestritten werden, dass eine Hire-and-FireGeschäftspolitik solchen Missbrauch reduzieren kann. Aber sollte man deshalb ein solches Arbeitsklima fordern, Mitbestimmungsrechte auf Null reduzieren und spricht das für die Diffamierung ganzer Beschäftigungsverhältnisse und Organisationseinheiten?

Diese Argumentation mit dem dahinter stehenden Menschenbild geht beispielsweise hinsichtlich der Privatisierungen in der Straffälligenhilfe auch deshalb fehl, weil sich in den Beiräten und Vorständen der privaten Träger, in deren Verbänden und Fachorganisationen hunderte von öffentlich Bediensteten finden, die diese Tätigkeiten zusätzlich, ehrenamtlich und engagiert verrichten.

Auch im wegen seiner Innovationsfreudigkeit zurecht hoch gelobten österreichischen Verein Neustart, vormals Verein für Bewährungshilfe und Soziale Arbeit, wurde über lange Zeit ein beachtlicher Teil der Leistung von auf Lebenszeit angestellten Beschäftigten erbracht, nicht von schlecht ausgebildeten Personen, die ständig um ihren Arbeitsplatz fürchten mussten. Insgesamt sollten internationale Erfahrungen nicht nur assoziativ und selektiv positiv für die Privatisierung zur Kenntnis und in Anspruch genommen werden. Sie müssen sorgfältig und kritisch daraufhin untersucht werden, inwiefern Erfolge wirklich hauptsächlich auf die privatwirtschaftlichen Steuerungsmethoden zurückzuführen sind. Hinsichtlich des Vereins Neustart sollte man dann auch weitere Arbeitsbedingungen zur Kenntnis nehmen, wie z. B. die auf 30 begrenzten Höchstfallzahlen, eine andere Tradition des Ehrenamtes, in der hoch professionelle Fachkräfte nicht gewinnorientiert tätig sind, das Zeugnisverweigerungs- recht und eine sehr aktive engagierte kriminalpolitische Öffentlichkeitsarbeit. Will man das mit übernehmen oder klaubt man sich etwas zusammen um zu sparen?

Insgesamt sollte die Debatte über die Privatisierung der Strafvollstreckung und insbesondere des Strafvollzugs auf eine seriöse und differenzierende Art (eben nicht marktschreierisch) geführt werden, um die

- politischen (Gerechtigkeit, Solidarität, demokratische Verantwortung und Kontrolle, Menschenbild, Ordnungspolitik, Sozialpolitik, Wettbewerbspolitik)

- wirtschaftlichen (Betriebswirtschaft, Volkswirtschaft) und

- rechtlichen (Verfassungsrecht, Strafvollzugsrecht, Haushaltsrecht) Aspekte

abwägen zu können und daraus Schlüsse zu ziehen. Das ideologische Behaupten einer Überlegenheit eines Steuerungsmodells bei gleichzeitigem Verstecken der Risiken und eigenen Interessen ist unseriös - möglicherweise ist es aber gegebenen Machtverhältnissen angemessen. Auf gute Argumente müssen wir dann noch weiter warten.

Allgemein spricht man seit über 30 Jahren von einer Dienstleistungsgesellschaft, in der Serviceleistungen im Mittelpunkt stehen und letztlich ist es inzwischen ein Gemeinplatz, dass es hinsichtlich der Wirtschaftlichkeit nicht bspw. um eine einzelne Fahrleistung per Auto oder Bahn, sondern die verkehrsmäßige Erschließung eines Wohn- und Arbeitsbereichs geht oder nicht um eine einzelne ärztliche Behandlungsleistung, sondern um Gesundheit. Sollten sich dann nicht auch alle Leistungsanbieter an das Vollzugsziel halten, das der Gesetzgeber einmütig in das Strafvollzugsgesetz geschrieben hat? Dort heißt es, dass im Vollzug der Freiheitsstrafe der Gefangene fähig werden soll, künftig in sozialer Verantwortung ein Leben ohne Straftaten zu führen. Output-Orientierung ist die Lösung der Zeit und ich schlage entsprechende Evaluationsforschungen 5 Jahre nach der Haftentlassung vor.

Noch hat sich kein privater Anbieter gefunden, der seine Leistungen nur dann bezahlt haben will, wenn er das Ziel erreicht hat.

Heinz Cornel ist Professor für Jugendrecht, Strafrecht und Kriminologie in Berlin und Mitherausgeber dieser Zeitschrift.

\section{Fußnoten}

1 Die freie Straffälligenhilfe beruhte schon immer auf privaten Initiativen, nicht aber auf Profitinteressen.

2 Der amerikanische Marktführer für Privatgefängnisse Corrections Corporation of America mit knapp 70000 Haftplätzen (Marktanteil etwa $55 \%$ ) konnte den Wert seiner Aktien innerhalb von 10 Jahren von 50 Millionen auf 3,5 Milliarden Dollar steigern, also versiebzigfachen. Da die Haftkosten staatlich garantiert sind, wird an der Börse mit dem Spruch für die Aktie geworben, dass die Firma einem Hotel gleiche, »das immer $\mathrm{zu}$ $100 \%$ belegt ....und bis zum Ende des Jahrhunderts ausgebucht ist.«

3 Vgl. Gasch, Ursula, Privatisierung des Strafvollzugs am Beispiel der USA, in: Bewährungshilfe 2004, S. 260ff., hier S. 268f.; Meyer, Frank, Privatisierung und Strafvollzug, in: Bewährungshilfe 2004, S. 272ff., hier S. 281 und Müller-Dietz in diesem Heft.

4 Maelicke, Bernd, Der Strafvollzug und die Neue Wirklichkeit, in; ZfStrVo 1999, S. 73ff., hier S.76.

5 Das war deutlich mehr als der Schaden, der im gleichen Jahr durch alle schweren Diebstähle entstand.

6 Immerhin handelte es sich aber um 111000 Insolvenzen im Jahr 2004 (davon 39000 Unternehmen) mit voraussichtlichen Forderungen von fast 40 Milliarden Euro.

7 »Das Einschließen von Menschen, das legale Entziehen der Freiheit ist die schärfste Sanktion, welche ein Staat seinen Bürgern zumuten kann. Freiheitsentzug gehört zum absoluten Kernbereich klassischen staatlichen Handelns.«, Flügge, Christoph, Das Geschäft mit der Sicherheit, in: ZfStrVo 2000, S.259ff, hier S. 261.

\section{GLOSSE ZUM THEMA}

Privatisierung ist prima. Privatisierung heißt: Jetzt kann wieder jeder alles selbst machen. Reine Privatsache zum Beispiel ist die neue Post. Die Briefkästen werden abgeschraubt, und man läuft selbst (mindestens 1 Stunde). Das nennt man dann einen kleinen Börsengang machen. Oder die PB, die Private Bahn AG. Strecken weg, Anschlüsse weg, Zug sowieso weg (falls er je kam). Besser, man fährt wieder ganz privat, im Auto. Klasse Idee auch von den Städten, ihre Wasserwerke zu verkaufen. Da wird bald jeder selbst nach einer Quelle graben. Und so ein kleines Klärwerk im Keller - warum nicht? Eine blutige Herausforderung für den praktischen Heimwerker dürfte schließlich die Komplettprivatisierung der städtischen Krankenhäuser sein, wie gerade in Hamburg diskutiert. Ein freies Bett für den Kassenpatienten? Alle zwei Jahre, wenn mal ein neues Medikament getestet wird. Bis dahin muss man eben selbst zur Säge greifen: Mutti, Tupfer! So richtig zur Sache geht es natürlich erst, wenn auch Polizei, Justiz und die Vollzugsanstalten privatisiert sind. Mit Privatknästen, wie einst auf den Ritterburgen am Rhein, so genannten Verliesen. Man kommt ins Träumen. Die Freiheit sieht erst wieder, wer, wie damals, ein kräftiges Lösegeld zahlt. Das belebt die Konjunktur, schon zieht die Binnennachfrage an. Kein Zweifel, Privatisierung bringt die Wirtschaft in Schwung.

Benedikt Erenz

Aus: Die Zeit vom 22. 5. 2003 